\title{
EQUIVALENCIA DE INGRESOS EN UN DUOPOLIO ELÉCTRICO*
}

\author{
Estrella Alonso \\ Universidad Pontificia Comillas, España \\ JUAN TEJADA \\ Universidad Complutense de Madrid, España
}

This article calculates the Bayes-Nash equilibria of a parametric family of auction models which includes, among others, the classic models. In an analysis of two power generation firms with the same production capacity and risk neutrality, the models in this class verify a revenue equivalence result.

JEL: C72, D44.

Keywords: Subastas, Equilibio Bayesiano de Nash, Mercado Eléctrico.

\section{INTRODUCCIÓN}

En las últimas décadas los mercados eléctricos de multitud de países alrededor del mundo han sido reestructurados y se aplica una subasta para regular el mercado eléctrico diario Fehr y Harbord $(1993,1998)^{1}$.

Cada empresa eléctrica oferta una cantidad de unidades de electricidad y un precio por unidad para cada una (o media) de las 24 horas del día siguiente. A partir de las ofertas se construye una lista de méritos, se ordenan los precios ofertados de menor a mayor hasta que quede completamente satisfecha la demanda. El precio de la última oferta necesaria para satisfacer la demanda se llama precio del mercado. Hay un organismo oficial, que llamaremos Operador del Mercado (subastador), que selecciona las mejores ofertas (las más bajas) hasta cubrir la demanda de ese día y de esa hora (o media). En la mayoría de los mercados eléctricos, el precio del mercado es el precio al que se realizan todas las transacciones. Este modelo de subasta se conoce con el nombre de modelo de subasta uniforme. Se ha discutido mucho, sin llegar a una conclusión

\footnotetext{
* Los autores están profundamente agradecidos a los árbitros anónimos y al editor de esta revista por su contribución a la mejora de este trabajo. Este trabajo se ha desarrollado bajo el soporte del proyecto MTM 2008-06778-C02-02.

E-mails: ealonso@upcomillas.es y jtejada@mat.ucm.es

${ }^{1}$ Para un conocimiento más profundo de la teoría de subastas y el diseño de mecanismos consultar: Milgrom (2004), Klemperer (1999) y Burguet (2000).
} 
clara (Ausubel y Cramton, 2002), sobre las ventajas y desventajas de este modelo frente a uno alternativo llamado modelo de subasta discriminatorio (Fabra, 2001, Fabra, Fehr y Harbord, 2002, y Fabra, Fehr y Harbord, 2006). En el modelo de subasta discriminatorio se paga a cada empresa generadora su propia puja. En la literatura encontramos artículos que argumentan a favor del modelo uniforme (Wolfram, 1999) y otros a favor del modelo discriminatorio (Federico y Rahman, 2001). Existen otros modelos de subasta que se emplean en contextos ajenos al mercado eléctrico como es el modelo de subasta de Vickrey (Vickrey, 1961).

El aporte principal de este trabajo es la agrupación, en una única expresión continua, de una familia de modelos de subasta que contiene como casos particulares a los modelos clásicos mencionados. Dicha expresión permite el cálculo simultáneo de equilibrios Bayesianos de Nash para todos los modelos. Es decir las empresas generadoras sabrán cuál es la oferta óptima para maximizar sus beneficios esperados que deben emplear bajo cualquiera de los modelos pertenecientes a la familia. Además, aunque nos vamos a centrar en un modelo de mercado simétrico, con idénticas capacidades de producción, costes independientes y empresas neutrales al riesgo (en cuyo caso obtendremos un resultado de equivalencia de ingresos para todos los modelos de subasta pertenecientes a la familia), al considerar diferentes hipótesis podemos encontrar nuevos modelos de subasta pertenecientes a la familia más ventajosos que cualquiera de los clásicos. Esto quedará demostrado en un ejemplo en las conclusiones.

La principal dificultad para analizar estos modelos de subasta reside en que los modelos de subasta del mercado eléctrico son modelos de subasta de múltiples objetos (se subasta el derecho a despachar la demanda eléctrica, que puede ser distribuida entre varias empresas) y el análisis de dichos modelos de subasta es muy complejo. En este trabajo se generaliza el Teorema de Equivalencia de Ingresos para subastas de un objeto (Myerson, 1981) a la subasta de múltiples objetos siguiente: la subasta de $m$ objetos idénticos más uno heterogéneo. Además, se verá que la familia de modelos de subasta considerada verifica las hipótesis de dicha generalización bajo determinadas circunstancias.

Existen otros trabajos donde se generaliza el teorema de equivalencia de ingresos. En Krishna (2002) se generaliza el teorema de equivalencia de ingresos a la subasta de m objetos idénticos. En Hinz (2004) se generaliza a la subasta eléctrica donde la demanda es estocástica pero los costes de producción son conocidos.

Por otra parte, en la mayoría de los artículos relacionados con la subasta de energía eléctrica, se modela la subasta empleada para las transacciones de compraventa como un juego no cooperativo de información completa (en el que los costes de producción de las empresas competidoras se consideran de conocimiento común).

Existen pocos artículos en los que se suponga que existe incertidumbre, por parte de una empresa, sobre la función de costes de las empresas contrarias:

- $\quad$ En Ferrero, Rivera y Shahidehpour (1998) se analiza un ejemplo numérico y discreto de un duopolio eléctrico bajo un modelo de subasta uniforme. Se 
considera, además, que las valoraciones son privadas pero correlacionadas. Los costes de producción son información privada de cada empresa, pero ambos dependientes del precio del combustible.

- $\quad$ En Bosco y Parisio (2001) se analiza un mercado eléctrico en el que participan $\mathrm{N}$ empresas, con dos unidades de producción cada una de ellas bajo un modelo de subasta uniforme. Se considera que las empresas son asimétricas en cuanto a capacidades de producción.

- $\quad$ En Schöne (2003) se analiza un modelo en duopolio eléctrico, con idénticas capacidades pero sólo bajo un modelo de subasta uniforme.

En contraste, en este artículo se considera que existe cierta incertidumbre sobre el coste de producción rival, es decir, se modela el mercado eléctrico como un juego de información incompleta.

El trabajo se estructura de la siguiente forma. En la Sección 2 se describe el modelo de mercado a considerar así como la familia de modelos de subasta a analizar. En la Sección 3 se calculan los Equilibrios Bayesianos de Nash para todos los modelos pertenecientes a la familia de modelos de subasta. En la Sección 4 se enuncia el resultado de Equivalencia de Ingresos y en la Sección 5 se dan las conclusiones obtenidas, incluyendo un ejemplo en el que se observa que un modelo nuevo perteneciente a la familia de modelos de subasta es más ventajoso que cualquiera de los tres clásicos.

\section{El Modelo}

Se van a suponer las siguientes hipótesis en el modelo de mercado:

- $\quad$ Hay dos empresas, $i \in\{1,2\}$, con sendas unidades de producción y neutrales al riesgo, interesadas en el despacho de unidades de electricidad en algún mercado eléctrico. Supondremos que ambas empresas tienen capacidades de producción idénticas $k=1$, siendo $k$ perfectamente divisible.

- $\quad$ La función de coste de la empresa $i, g\left(q_{i}, \theta_{i}\right)$, es creciente y diferenciable en ambas variables, donde $q_{i} \in[0,1]$ es la cantidad generada por $i \in\{1,2\}$. Además $g\left(0, \theta_{i}\right)=0$.

- $\quad$ El tipo, $\theta_{i}$ es una realización independiente de una variable aleatoria continua $\Theta_{i}$ con función de distribución $F$ acotada en $\Re$ y absolutamente continua, con función de densidad $f$ en el intervalo $\left[a_{1}, a_{2}\right]$. El jugador $i \mathrm{y}$ sólo el jugador $i$, observa la realización $\theta_{i}$, modelizando de esta manera la incertidumbre que el jugador $j$ tiene acerca del coste de producción de la empresa $i$.

- $\quad D$ denota la demanda eléctrica de un determinado periodo y se considera inelástica al precio.

- $\quad$ Cada empresa $i \in\{1,2\}$, independiente y simultáneamente, realiza una oferta $b_{i} \in\left[0, b_{\max }\right]$, donde especifica el mínimo precio por unidad al que está dispuesta a vender la totalidad de su capacidad. 
Una estrategia para la empresa $i \in\{1,2\}$ es, entonces, una función de oferta de la forma $b_{i}\left(\theta_{i}\right):\left[a_{1}, a_{2}\right] \rightarrow\left[0, b_{\max }\right]$. El valor real positivo $b_{\max }$ es el precio máximo que el Operador del Mercado está dispuesto a pagar por cada unidad eléctrica necesaria para satisfacer la demanda eléctrica $D$. El precio $b_{\max }$ es tal que el Operador del Mercado pueda asegurar a las empresas que entren en el mercado, al menos el coste de producción de cualquiera de sus unidades eléctricas.

Por la simetría del problema, se puede conjeturar que, en equilibrio, las dos empresas se comporten de la misma manera estratégica y, por tanto, limitaremos nuestra búsqueda a equilibrios Bayesianos de Nash simétricos. Luego supondremos que $b_{i}\left(\theta_{i}\right)=b\left(\theta_{i}\right)$ es la función de oferta utilizada en equilibrio por los dos jugadores, donde $b\left(\theta_{i}\right)$ es una función estrictamente creciente y diferenciable.

A este modelo de mercado, verificando las hipótesis enunciadas, lo llamaremos Modelo Simétrico Inicial.

Con el fin de satisfacer la demanda, el Operador del Mercado ordena las ofertas de menor a mayor, de tal forma que la empresa de menor oferta despacha primero. Si su capacidad no es suficiente para satisfacer la demanda, la empresa de mayor oferta despacha la demanda residual, siempre y cuando ésta sea inferior a su propia capacidad. Luego la cantidad que despacha la empresa $i \in\{1,2\}$ está dada por la siguiente función:

$$
Q_{i}\left(b_{1}, b_{2}\right)=\left\{\begin{array}{lll}
\min (1, D) & \text { si } & b_{i}<b_{j} \\
0 & \text { si } & b_{i}>b_{j} \text { y } D \leq 1 \\
\min (D-1,1) & \text { si } & b_{i}>b_{j} \text { y } D>1
\end{array}\right.
$$

Todos los aspectos de este juego Bayesiano, además del modelo de subasta concreto que se va a utilizar para las transacciones del mercado eléctrico, son de conocimiento común.

El precio fijado para la compra de unidades eléctricas depende del modelo de subasta que se esté empleando para realizar las transacciones. Hay tres modelos de subasta clásicos aplicables al mercado eléctrico: modelo de subasta uniforme, modelo de subasta discriminatoria y modelo de subasta de Vickrey. Estos han sido analizados ampliamente pero con costes conocidos. Describamos brevemente cómo se fija el precio de compra en cada uno de ellos:

En el modelo uniforme, el precio que el Operador del Mercado paga a cada empresa por unidad eléctrica suministrada es igual a la mayor oferta aceptada. Todas las empresas que despachan en el mercado reciben el mismo precio por unidad eléctrica.

En el modelo discriminatorio, el precio que el Operador del Mercado paga a cada empresa por unidad eléctrica suministrada es igual a su propia oferta. Las empresas que despachan en el mercado reciben precios distintos por unidad eléctrica.

En el modelo de Vickrey, la regla mediante la cual el Operador del Mercado establece el precio es algo más complicada. A cada empresa le pagan, 
por cada unidad de electricidad despachada, el precio correspondiente a la unidad que desplaza. Es decir, el precio de la unidad eléctrica necesaria para seguir cubriendo la demanda en el caso de que la primera empresa retirase su oferta del mercado. En el caso de que la empresa de mayor oferta sea necesaria para satisfacer parte de la demanda, a ésta se le pagaría el precio máximo $b_{\max }$ por unidad despachada. En particular, supongamos que hay dos empresas y que la empresa $i$ es la empresa de menor oferta (por tanto, la que entra primero en el mercado) entonces le pagan la oferta contraria $b_{j}$ si $D \leq 1$. Y si $D>1$ dicha empresa $i$, de menor oferta, recibe dos precios distintos por las unidades despachadas: la oferta contraria $b_{j}$ por cada una de las $D-1$ primeras unidades y la oferta máxima permitida $b_{\max }$ por cada una de las $2-D$ unidades restantes. A la empresa de mayor oferta le pagan 0 si $D \leq 1$ y $b_{\max }$ por cada una de las unidades que despacha si $D>1$.

En este artículo no limitaremos nuestro análisis a los tres modelos clásicos ya que se analizará una familia paramétrica de modelos de subasta, que contiene a los tres modelos clásicos como casos particulares. Dicha familia tendrá asociada la siguiente función de beneficio de la empresa $i \in\{1,2\}$ :

$$
B_{i}\left(\theta_{i}, b_{i}, b_{j}\right)=\left\{\begin{array}{lll}
\gamma_{1} b_{i}+\beta_{1} b_{j}+\varphi b_{\max }-g\left(\phi_{1}, \theta_{i}\right) & \text { si } b_{i}<b_{j} \\
\gamma_{2} b_{i}+\varphi b_{\max }-g\left(\phi_{2}, \theta_{i}\right) & \text { si } b_{i}>b_{j}
\end{array}\right.
$$

con $j \in\{1,2\}, i \neq j$, siendo $\gamma_{1}, \beta_{1}, \varphi, \gamma_{2} \in[0, \infty), \gamma_{1}+\beta_{1}+\varphi=\phi_{1},+\gamma_{2}+\varphi=\phi_{2}$ y donde $\phi_{1}$ y $\phi_{2}$ están determinados por la demanda. En esta función se está considerando que, si la empresa $i$ oferta la menor de las dos pujas, la cantidad que despachará la empresa $i$ es $\gamma_{1}+\beta_{1}+\varphi=\phi_{1}$, recibiendo posiblemente distintos precios por ese total de unidades eléctricas despachadas. Es decir, el Operador del Mercado paga a $i$, si ésta realiza la menor de las pujas, $\gamma_{1}$ unidades al precio unitario $b_{i}, \beta_{1}$ unidades al precio unitario $b_{j}$ y $\varphi$ unidades al precio unitario $b_{\max }$. Por otra parte, si la empresa $i$ realiza la mayor de las pujas, despachará un total de $\gamma_{2}+\varphi=\phi_{2}$ unidades y el Operador del Mercado paga a $i, \gamma_{2}$ unidades al precio unitario $b_{1}$ y $\varphi$ unidades al precio unitario $b_{\text {max }}$. Además, si la demanda $D$ es menor o igual que 1 , entonces $\phi_{1}=D$ y $\phi_{2}=0$. Si la demanda verifica $D=1+\alpha$, con $\alpha \in(0,1)$, entonces $\phi_{1}=1$ y $\phi_{2}=\alpha$. Si la demanda es mayor o igual que 2 , entonces $\phi_{1}=1$ y $\phi_{2}=1$.

Esta familia de modelos de subasta verifica dos principios exigibles en una subasta eléctrica, que son:

- $\quad$ La puja que una empresa hace es el mínimo precio al que está dispuesta a vender toda su capacidad. Una vez realizadas las pujas, el Operador del Mercado no puede pagar a una empresa generadora un precio menor que su propia puja.

- Debe entrar en el mercado en primer lugar la empresa de menor oferta y, si ésta no es capaz de satisfacer la totalidad de la demanda, debe entrar la otra empresa para despachar la demanda residual. 
La familia paramétrica de modelos de subasta que hemos introducido la denominaremos Modelo de Subasta General y se denotará por MSG.

Evidentemente, la asignación de valores a los parámetros fija las funciones de beneficio y el modelo de subasta empleado para la transacción queda completamente determinado. Como el modelo de subasta a utilizar es de conocimiento común, el Operador del Mercado anuncia a priori el modelo que va a utilizar para pagar a las empresas generadoras. El rango de los posibles valores de los parámetros dependerá del tamaño de la demanda respecto del de las capacidades. Por ello, se distinguirán los siguientes casos:

\section{Caso 1: Demanda satisfecha por una empresa}

Las dos empresas tienen capacidad suficiente para satisfacer la demanda por sí sola, es decir, $D \leq 1$. En este caso, la empresa que oferta el menor precio despachará toda la demanda y la otra empresa no despachará ninguna unidad eléctrica, es decir, quedará fuera del mercado. Luego, sustituyendo en la expresión $\$ 2.1$. $\$$ los valores $\phi_{1}=D, \phi_{2}=0, \gamma_{2}=\varphi=0$ y $\beta_{1}=D-\gamma_{1}$, la familia de modelos MSG, en este Caso 1, queda reducida a la siguiente familia uniparamétrica con beneficio

$$
B_{i}^{\gamma_{1}}\left(\theta_{i}, b_{i}, b_{j}\right)=\left\{\begin{array}{ccc}
\gamma_{1} b_{i}+\left(D-\gamma_{1}\right) b_{j}-g\left(D, \theta_{i}\right) & \text { si } & b_{i}<b_{j} \\
0 & \text { si } & b_{i}>b_{j}
\end{array} \quad \gamma_{1} \in[0, \mathrm{D}] .\right.
$$

En particular, si $\gamma_{1}=D$, se obtiene el modelo uniforme y discriminatorio, que coinciden cuando la demanda es menor o igual que la capacidad de producción de cualquiera de las dos empresas. Si $\gamma_{1}=0$ se obtiene el modelo de Vickrey.

\section{Caso 2: Demanda no satisfecha por una empresa pero sí por dos}

Se necesita la capacidad de ambas empresas para satisfacer la demanda, pero ésta es menor que la suma de las dos capacidades, es decir, $1<D=1+\alpha<2$, donde $\alpha \in(0,1)$. En este caso, la empresa que oferta el menor precio despachará toda su capacidad 1 y la otra empresa despachará la demanda residual $D-1=\alpha$. Luego sustituyendo en la expresión \$2.1. \$ los valores $\phi_{1}=1, \phi_{2}=\alpha, \varphi=\alpha-\gamma_{2}$ y $\beta_{1}=1-\alpha-\gamma_{1}+\gamma_{2}$, la familia MSG, en este Caso 2, queda reducida a la siguiente familia biparamétrica con beneficio

$$
B_{i}^{\left(\gamma_{1}, \gamma_{2}\right)}\left(\theta_{i}, b_{i}, b_{j}\right)=\left\{\begin{array}{ccc}
\gamma_{1} b_{i}+\left(1-\alpha-\gamma_{1}+\gamma_{2}\right) b_{j} & \text { si } & b_{i}<b_{j} \\
+\left(\alpha-\gamma_{2}\right) b_{\max }-g\left(1, \theta_{i}\right) & & \\
\gamma_{2} b_{i}+\left(\alpha-\gamma_{2}\right) b_{\max }-g\left(\alpha, \theta_{i}\right) & \text { si } & b_{i}>b_{j}
\end{array}\right.
$$

donde $\gamma_{1} \in\left[0,1-\alpha+\gamma_{2}\right]$ y $\gamma_{2} \in[0, \alpha]$. 
En particular, si $\gamma_{1}=0$ y $\gamma_{2}=\alpha$, se obtiene el modelo uniforme. Si $\gamma_{1}=1$ y $\gamma_{2}=\alpha$, se obtiene el modelo discriminatorio. Por último, si $\gamma_{1}=0 \mathrm{y}$ $\gamma_{2}=0$, se obtiene el modelo de Vickrey.

\section{Caso 3: Demanda no satisfecha por las dos empresas}

La demanda excede la suma de las dos capacidades, es decir $2 \leq D$. Ambas empresas despachan la totalidad de sus capacidades. En este caso no hay competencia y las empresas pujarán la máxima oferta permitida $b_{\max }$. Es un caso que sólo consideraremos como caso límite del anterior, a efectos de continuidad, ya que bajo cualquier modelo de subasta las empresas ingresan con seguridad $b_{\max }$ y el Operador del Mercado pagará $2 b_{\max }$.

\section{Equilibrios Bayesianos de Nash bajo el Modelo de Subasta GenERAL}

El siguiente resultado da la expresión del equilibrio Bayesiano de Nash para cada uno de los modelos pertenecientes a MSG y por tanto lestrategia a seguir por cada empresa si desea maximizar su beneficio esperado.

Proposición 1: Si se verifican las hipótesis del Modelo Simétrico Inicial se utiliza un modelo de subasta $\mathcal{S} \in \mathrm{MSG}$, es decir, la función de beneficio de la empresa $i \in\{1,2\}$ es de la forma:

$$
B_{i}\left(\theta_{i}, b_{i}, b_{j}\right)=\left\{\begin{array}{ccc}
\gamma_{1} b_{i}+\beta_{1} b_{j}+\varphi b_{\max }-g\left(\phi_{1}, \theta_{i}\right) & \text { si } & b_{i}<b_{j} \\
\gamma_{2} b_{i}+\varphi b_{\max }-g\left(\phi_{2}, \theta_{i}\right) & \text { si } & b_{i}>b_{j}
\end{array}\right.
$$

$\operatorname{con} j \in\{1,2\}, i \neq j, \gamma_{1}, \beta_{1}, \varphi, \gamma_{2} \in[0, \infty), \gamma_{1}+\beta_{1}+\varphi=\phi_{1},+\gamma_{2}+\varphi=\phi_{2}$, $\phi_{1}+\phi_{2}=D$ y $D<2$, entonces:

Región I: Si $\gamma_{2} \neq 0$, se verifica que existe un único equilibrio Bayesiano de Nash simétrico $\left(b^{*}\left(\theta_{1}\right), b^{*}\left(\theta_{2}\right)\right)$, cumpliendo la condición $b^{*}\left(a_{2}\right)=b_{\max }$ con $b_{\max } \geq\left(g\left(\phi_{1}, a_{2}\right)-g\left(\phi_{2}, a_{2}\right)\right) /\left(\phi_{1}-\phi_{2}\right)$ que viene dado por la siguiente expresión:

$$
\begin{aligned}
b^{*}\left(\theta_{i}\right)= & \frac{g\left(\phi_{1}, \theta_{i}\right)-g\left(\phi_{2}, \theta_{i}\right)}{\phi_{1}-\phi_{2}}+\left(\gamma_{1}-\left(\gamma_{1}-\gamma_{2}\right) F\left(\theta_{i}\right)\right)^{-\frac{\phi_{1}-\phi_{2}}{\gamma_{1}-\gamma_{2}}}\left(b_{\max } \gamma_{2}^{\frac{\phi_{1}-\phi_{2}}{\gamma_{1}-\gamma_{2}}}+\right. \\
& \frac{1}{\phi_{1}-\phi_{2}} \int_{\theta_{i}}^{a_{2}} \frac{\partial}{\partial t}\left(g\left(\phi_{1}, t\right)-g\left(\phi_{2}, t\right)\right)\left(\gamma_{1}-\left(\gamma_{1}-\gamma_{2}\right) F(t)\right)^{\frac{\phi_{1}-\phi_{2}}{\gamma_{1}-\gamma_{2}}} d t \\
& \left.-\frac{g\left(\phi_{1}, a_{2}\right)-g\left(\phi_{2}, a_{2}\right)}{\phi_{1}-\phi_{2}} \gamma_{2}^{\frac{\gamma \phi_{1}-\phi_{2}}{\gamma_{1}-\gamma_{2}}}\right)
\end{aligned}
$$

si $\gamma_{1} \neq \gamma_{2}, y$ 


$$
\begin{aligned}
b^{*}\left(\theta_{i}\right)= & \frac{g\left(\phi_{1}, \theta_{i}\right)-g\left(\phi_{2}, \theta_{i}\right)}{\phi_{1}-\phi_{2}} \\
& +e^{-\frac{\phi_{1}-\phi_{2}}{\gamma_{1}}\left(1-F\left(\theta_{i}\right)\right)}\left(b_{\max }-\frac{g\left(\phi_{1}, a_{2}\right)-g\left(\phi_{2}, a_{2}\right)}{\phi_{1}-\phi_{2}}\right) \\
& +\frac{e^{\frac{\phi_{1}-\phi_{2}}{\gamma_{1}}\left(1-F\left(\theta_{i}\right)\right)}}{\phi_{1}-\phi_{2}} \int_{\theta_{i}}^{a_{2}} \frac{\partial}{\partial t}\left(g\left(\phi_{1}, t\right)-g\left(\phi_{2}, t\right)\right) e^{-\frac{\phi_{1}-\phi_{2}}{\gamma_{1}}(1-F(t))} d t
\end{aligned}
$$

si $\gamma_{1}=\gamma_{2}$.

Región II: $\operatorname{Si} \gamma_{2}=0$ y $\gamma_{1}=0$, se verifica que existe una única estrategia dominante para cada empresa $i \in\{1,2\}$ dada por:

$$
b^{*}\left(\theta_{i}\right)=\frac{g\left(\phi_{1}, \theta_{i}\right)-g\left(\phi_{2}, \theta_{i}\right)}{\phi_{1}-\phi_{2}} .
$$

Región III: $\operatorname{Si} \gamma_{2}=0$ y $\gamma_{1} \neq 0$, se verifica que el único equilibrio Bayesiano de Nash simétrico $\left(b^{*}\left(\theta_{1}\right), b^{*}\left(\theta_{2}\right)\right)$ viene dado por:

$$
\begin{aligned}
b^{*}\left(\theta_{i}\right)= & \frac{g\left(\phi_{1}, \theta_{i}\right)-g\left(\phi_{2}, \theta_{i}\right)}{\phi_{1}-\phi_{2}} \\
& +\frac{\left(1-F\left(\theta_{i}\right)\right)^{-\frac{\phi_{1}-\phi_{2}}{\gamma_{1}}}}{\phi_{1}-\phi_{2}} \int_{\theta_{i}}^{a_{2}} \frac{\partial}{\partial t}\left(g\left(\phi_{1}, t\right)-g\left(\phi_{2}, t\right)\right)(1-F(t))^{\frac{\phi_{1}-\phi_{2}}{\gamma_{1}}} d t .
\end{aligned}
$$

Demostración: En el apéndice.

ObSERVACión 2: En la Región II y en la Región III se verifica que $b^{*}\left(a_{2}\right)=\left(g\left(\phi_{1}, a_{2}\right)-g\left(\phi_{2}, a_{2}\right)\right) /\left(\phi_{1}-\phi_{2}\right)$, sin necesidad, en ambos casos, de imponer ninguna condición. Se debe a que $\gamma_{2}=0$, en cuyo caso el precio de la demanda residual no queda fijado por la propia oferta (obsérvese la expresión (1)) y, por tanto, la competencia es extrema por entrar primero al mercado. Dicho de otra forma, la demanda residual o no existe o tiene un precio fijo independiente de la propia oferta, la competencia es extrema por el tramo inicial. Bajo esas circunstancias, las ofertas no se disparan y no es necesario, por tanto, que el Operador del Mercado fije una puja máxima $b_{\max }$. Sin embargo, en la Región I el Operador del Mercado debe imponer una oferta máxima. En efecto, si $\gamma_{2} \neq 0$ las dos empresas entran con total seguridad en el mercado y si una empresa tiene un tipo alto, se ve tentada a pujar lo máximo posible al estar segura de entrar en el mercado despachando, al menos, la demanda 
residual en la que ahora si puede fijar precio. Por otro lado, si la demanda residual tiende a 1, las empresas se ven tentadas a pujar por la demanda residual el mayor precio permitido. De ahí la importancia de que en este tipo de mercados se fije una puja máxima. Es evidente que, cuanto mayor sea $b_{\text {max }}$, mayor será la puja realizada por las empresas, en cualquiera de los modelos de la Región I. Esto hace que al Operador del Mercado le interese fijar $b_{\max }$ lo mínimo posible pero asegurando a las empresas, al menos el coste de producción de cualquier unidad eléctrica, es decir, le interesa fijar $b_{\max }=\left(g\left(\phi_{1}, a_{2}\right)-g\left(\phi_{2}, a_{2}\right)\right) /\left(\phi_{1}-\phi_{2}\right)$.

Corolario 3: Si se verifican las hipótesis del Modelo Simétrico Inicial, $D<2$ y se utiliza un modelo de subasta $\mathcal{S} \in$ MSG donde $b_{\max }=\left(g\left(\phi_{1}, a_{2}\right)-g\left(\phi_{2}, a_{2}\right)\right) /$ $\left(\phi_{1}-\phi_{2}\right)$, entonces existe un único equilibrio Bayesiano de Nash simétrico $\left(b^{*}\left(\theta_{1}\right), b^{*}\left(\theta_{2}\right)\right)$ verificando $b^{*}\left(a_{2}\right)=\left(g\left(\phi_{1}, a_{2}\right)-g\left(\phi_{2}, a_{2}\right)\right) /\left(\phi_{1}-\phi_{2}\right), \mathrm{y}$ dado por la siguiente expresión:

$$
\begin{aligned}
b^{*}\left(\theta_{i}\right)= & \frac{g\left(\phi_{1}, \theta_{i}\right)-g\left(\phi_{2}, \theta_{i}\right)}{\phi_{1}-\phi_{2}}+ \\
& \frac{\left(\gamma_{1}-\left(\gamma_{1}-\gamma_{2}\right) F\left(\theta_{i}\right)\right)^{-\frac{\phi_{1}-\phi_{2}}{\gamma_{1}-\gamma_{2}}}}{\phi_{1}-\phi_{2}} \int_{\theta_{i}}^{a_{2}} \frac{\partial}{\partial t}\left(g\left(\phi_{1}, t\right)\right. \\
& \left.-g\left(\phi_{2}, t\right)\right)\left(\gamma_{1}-\left(\gamma_{1}-\gamma_{2}\right) F(t)\right)^{\frac{\phi_{1}-\phi_{2}}{\gamma_{1}-\gamma_{2}}} d t
\end{aligned}
$$

si $\gamma_{1} \neq \gamma_{2}$

$$
\begin{aligned}
& b^{*}\left(\theta_{i}\right)=\frac{g\left(\phi_{1}, \theta_{i}\right)-g\left(\phi_{2}, \theta_{i}\right)}{\phi_{1}-\phi_{2}}+\frac{e^{\frac{\phi_{1}-\phi_{2}}{\gamma_{1}}\left(1-F\left(\theta_{i}\right)\right)}}{\phi_{1}-\phi_{2}} \int_{\theta_{i}}^{a_{2}} \frac{\partial}{\partial t}\left(g\left(\phi_{1}, t\right)\right. \\
& \left.-g\left(\phi_{2}, t\right)\right) e^{-\frac{\phi_{1}-\phi_{2}}{\gamma_{1}}(1-F(t))} d t \\
& \text { si } \gamma_{1}=\gamma_{2} \neq 0 \text { y } \\
& b^{*}\left(\theta_{i}\right)=\frac{g\left(\phi_{1}, \theta_{i}\right)-g\left(\phi_{2}, \theta_{i}\right)}{\phi_{1}-\phi_{2}} \\
& \text { si } \gamma_{1}=\gamma_{2}=0 \text {. }
\end{aligned}
$$

Demostración: En el apéndice. 
Realmente las expresiones que vamos a utilizar son la de los equilibrios Bayesianos de Nash en los siguientes casos particulares:

Caso 1: Demanda satisfecha por una empresa $(D \leq 1)$

En este caso, $\phi_{1}=D, \phi_{2}=0, \gamma_{2}=\varphi=0$ y $\beta_{1}=D-\gamma_{1}$. Por el Corolario 3 se obtiene:

i) $\quad$ Si $\gamma_{1}=0, b^{*}\left(\theta_{i}\right)=\left(g\left(D, \theta_{i}\right) / D\right.$.

ii) $\quad$ Si $\gamma_{1} \neq 0$,

$b^{*}\left(\theta_{i}\right)=\frac{1}{D}\left(g\left(D, \theta_{i}\right)+\left(1-F\left(\theta_{i}\right)\right)^{-\frac{D}{\gamma_{1}}} \int_{\theta_{i}}^{a_{2}} \frac{\partial}{\partial t} g(D, t)(1-F(t))^{\frac{D}{\gamma_{1}}} d t\right)$.

Caso 2: Demanda no satisfecha por una empresa pero sí por dos $(1<D=1+\alpha<2)$

En este caso, $\phi_{1}=1, \phi_{2}=\alpha, \varphi=\alpha-\gamma_{2}$ y $\beta=1-\alpha-\gamma_{1}+\gamma_{2}$. Por el Corolario 3 se obtiene:

i) $\quad$ Si $\gamma_{1} \neq \gamma_{2}$ :

$$
\begin{aligned}
b^{*}\left(\theta_{i}\right)= & \frac{g\left(1, \theta_{i}\right)-g\left(\alpha, \theta_{i}\right)}{1-\alpha}+\frac{\left(\gamma_{1}-\left(\gamma_{1}-\gamma_{2}\right) F\left(\theta_{i}\right)\right)^{-\frac{1-\alpha}{\gamma_{1}-\gamma_{2}}}}{1-\alpha} \\
& \left.\int_{\theta_{i}}^{a_{2}} \frac{\partial}{\partial t}(g(1, t)-g(\alpha, t))\left(\gamma_{1}-\left(\gamma_{1}-\gamma_{2}\right) F(t)\right)^{\frac{1-\alpha}{\gamma_{1}-\gamma_{2}}} d t\right) .
\end{aligned}
$$

ii) $\quad$ Si $\gamma_{1}=\gamma_{2} \neq 0$ :

$$
\begin{aligned}
b^{*}\left(\theta_{i}\right)= & \frac{g\left(1, \theta_{i}\right)-g\left(\alpha, \theta_{i}\right)}{1-\alpha} \\
& +\frac{e^{\frac{1-\alpha}{\gamma_{1}}\left(1-F\left(\theta_{i}\right)\right)}}{1-\alpha} \int_{\theta_{i}}^{a_{2}} \frac{\partial}{\partial t}(g(1, t)-g(\alpha, t)) e^{-\frac{1-\alpha}{\gamma_{1}}(1-F(t))} d t .
\end{aligned}
$$

iii) $\quad$ Si $\gamma_{1}=\gamma_{2}=0, b^{*}\left(\theta_{i}\right)=\left(g\left(1, \theta_{i}\right)-g\left(\alpha, \theta_{i}\right)\right) /(1-\alpha)$. 
OBSERVACión 4: Una vez calculados los equilibrios Bayesianos de Nash, podemos calcular el ingreso esperado por las empresas y el pago que espera realizar el Operador del Mercado. Se comprueba fácilmente que dichas expresiones son independientes de los parámetros que aparecen en MSG. Es decir, se verifica un resultado de Equivalencia de Ingresos bajo cualquiera de los modelos de subasta pertenecientes a MSG. Es más, hemos demostrado un resultado de Equivalencia de Ingresos más general.

\section{Teorema de Equivalencia de Ingresos en El mercado ELÉCTRICO.}

En esta sección se generalizará el Teorema de Equivalencia de Ingresos \} para subastas de un objeto (Myerson, 1981) a la subasta de múltiples objetos siguiente: la subasta de $m$ objetos idénticos más uno heterogéneo. Se demostrará que bajo dos modelos de subasta con $n$ empresas, verificándose las hipótesis del Modelo Simétrico Inicial, cada empresa obtiene el mismo ingreso esperado y, por tanto, el subastador espera pagar lo mismo por la demanda eléctrica, siempre y cuando, estos dos modelos de subasta verifiquen que dan el mismo ingreso esperado a las dos empresas, cuando su tipo es máximo.

Ahora una empresa puede "ganar" el objeto heterogéneo (el despacho de la demanda residual $\alpha$ ) o puede "ganar" uno de los objetos homogéneos (el despacho de toda su capacidad). El modelo de mercado que se va a considerar, en la primera parte de la presente sección, verificará todas las hipótesis del Modelo Simétrico Inicial pero, con $n$ empresas, en lugar de 2.

Si la demanda es $D=m+\alpha$, con $m \in\{0,1,2, \ldots, n-1\}$ y $\alpha \in[0,1)$, habrá $m$ empresas que podrán generar toda su capacidad (las $m$ menores ofertas) y una empresa que generará la demanda residual de $\alpha$ unidades (la oferta siguiente). El resto de empresas, $n-(m+1)$, ni ganará ni pagará nada.

El conjunto de modelos de subasta MSG que consideramos en el Modelo Simétrico inicial está incluido en un conjunto de modelos de subasta más amplio que describimos en la siguiente definición:

Definición 5: Denominaremos conjunto de modelos de subasta eléctrica y se denotará por $\mathcal{S E}$ al conjunto de modelos de subasta verificando lo siguiente:

i) Existe un único equilibrio Bayesiano de $\operatorname{Nash}\left(b^{*}\left(\theta_{1}\right), \ldots, b^{*}\left(\theta_{n}\right)\right)$ con $b^{*}\left(\theta_{i}\right)$ función estrictamente creciente y diferenciable.

ii) La regla de asignación de un modelo de subasta eléctrica $S \in \mathcal{S E}$ es de la forma:

$$
\left\{\omega_{i}^{S}\left(b_{1}, \ldots, b_{n}\right), \rho_{i}^{S}\left(b_{1}, \ldots, b_{n}\right), \psi_{i}^{S}\left(b_{1}, \ldots, b_{n}\right), \chi_{i}^{S}\left(b_{1}, \ldots, b_{n}\right)\right\}_{i=1}^{n}
$$

donde $\omega_{i}^{S}\left(b_{1}, \ldots, b_{n}\right)$ es el ingreso que obtiene la empresa $i$ si genera en el mercado la demanda residual; $\rho_{i}^{S}\left(b_{1}, \ldots, b_{n}\right)$ es el ingreso que obtiene 
la empresa $i$ si genera en el mercado toda su capacidad; $\psi_{i}^{S}\left(b_{1}, \ldots\right.$, $\left.b_{n}\right)=\operatorname{Prob}\left(b_{i}=b_{(m+1)}\right)$ es la probabilidad de que la empresa $i$ despache la demanda residual y, por último, $\chi_{i}^{S}\left(b_{1}, \ldots, b_{n}\right)=\operatorname{Prob}\left(b_{i} \leq b_{(m)}\right)$ es la probabilidad de que la empresa $i$ despache toda su capacidad, si las pujas son $b_{1}, \ldots, b_{n}$.

La función de beneficio del jugador $i$ en un modelo $S \in \mathcal{S E}$ es:

$$
\begin{aligned}
B_{i}\left(b_{1}, \ldots, b_{n} ; \theta_{i}\right)= & \left(\omega_{i}^{S}\left(b_{1}, \ldots, b_{n}\right)-g\left(\alpha, \theta_{i}\right) \psi_{i}^{S}\left(b_{1}, \ldots, b_{n}\right)\right. \\
& +\left(\rho_{i}^{S}\left(b_{1}, \ldots, b_{n}\right)-g\left(1, \theta_{i}\right)\right) \chi_{i}^{S}\left(b_{1}, \ldots, b_{n}\right) .
\end{aligned}
$$

Obsérvese que lo que diferencia dos modelos pertenecientes a $\mathcal{S E}$ es, en realidad, $\omega_{i}^{S}\left(b_{1}, \ldots, b_{n}\right)$ y $\rho_{i}^{S}\left(b_{1}, \ldots, b_{n}\right)$, ya que las otras dos componentes de la regla de asignación coinciden en todo modelo $S \in \mathcal{S E}$. Si evaluamos la regla de asignación en el equilibrio se obtiene:

$$
\begin{gathered}
\left\{\omega_{i}^{S}\left(b^{*}\left(\theta_{1}\right), \ldots, b^{*}\left(\theta_{n}\right)\right), \rho_{i}^{S}\left(b^{*}\left(\theta_{1}\right), \ldots, b^{*}\left(\theta_{n}\right)\right),\right. \\
\left.\psi_{i}^{S}\left(b^{*}\left(\theta_{1}\right), \ldots, b^{*}\left(\theta_{n}\right)\right), \chi_{i}^{S}\left(b^{*}\left(\theta_{1}\right), \ldots, b^{*}\left(\theta_{n}\right)\right)\right\}_{i=1}^{n} \\
=\left\{\tilde{\omega}_{i}\left(\theta_{1}, \ldots, \theta_{n}\right), \tilde{\rho}_{i}\left(\theta_{1}, \ldots, \theta_{n}\right), \tilde{\psi}_{i}\left(\theta_{1}, \ldots, \theta_{n}\right), \tilde{\chi}_{i}\left(\theta_{1}, \ldots, \theta_{n}\right)\right\}_{i=1}^{n},
\end{gathered}
$$

donde $\tilde{\omega}_{i}\left(\theta_{1}, \ldots, \theta_{n}\right)$ es el ingreso que obtiene la empresa $i$ si genera en el mercado la demanda residual, $\tilde{\rho}_{i}\left(\theta_{1}, \ldots, \theta_{n}\right)$ es el ingreso que obtiene la empresa $i$ si genera en el mercado toda su capacidad; $\tilde{\psi}_{i}\left(\theta_{1}, \ldots, \theta_{n}\right)$ es la probabilidad de que la empresa $i$ despache la demanda residual y, finalmente, $\tilde{\chi}_{i}\left(\theta_{1}, \ldots, \theta_{n}\right)$ es la probabilidad de que la empresa $i$ despache toda su capacidad, si los tipos son $\theta_{1}, \ldots, \theta_{n}$.

Por otro lado denotaremos por:

$$
\begin{aligned}
q_{i}^{S}\left(\theta_{i}\right) & =E_{\Theta_{-i}}\left[\tilde{\omega}_{i}\left(\theta_{1}, \ldots, \theta_{n}\right) \tilde{\psi}_{i}\left(\theta_{1}, \ldots, \theta_{n}\right)\right] \\
p_{i}^{S}\left(\theta_{i}\right) & =E_{\Theta_{-i}}\left[\tilde{\rho}_{i}\left(\theta_{1}, \ldots, \theta_{n}\right) \tilde{\chi}_{i}\left(\theta_{1}, \ldots, \theta_{n}\right)\right] \\
y_{i}^{S}\left(\theta_{i}\right) & =E_{\Theta_{-i}}\left[\tilde{\psi}_{i}\left(\theta_{1}, \ldots, \theta_{n}\right)\right] \\
z_{i}^{S}\left(\theta_{i}\right) & =E_{\Theta_{-i}}\left[\tilde{\chi}_{i}\left(\theta_{1}, \ldots, \theta_{n}\right)\right]
\end{aligned}
$$

al ingreso esperado por la empresa $i$ si genera en el mercado la demanda residual, al ingreso esperado por la empresa $i$ si genera en el mercado toda su capacidad, a la probabilidad esperada de que la empresa $i$ genere la demanda residual $\alpha$ y a la probabilidad esperada de que la empresa $i$ genere toda su capacidad, si su tipo es $\theta_{i}$, respectivamente. Obsérvese que el ingreso total esperado por la empresa $i, P_{i}^{S}\left(\theta_{i}\right)$ si genera en el mercado, es la suma: 
$P_{i}^{S}\left(\theta_{i}\right)=p_{i}^{S}\left(\theta_{i}\right)+q_{i}^{S}\left(\theta_{i}\right)$

Téngase en cuenta que siempre se verifica $y_{i}^{S_{1}}\left(\theta_{i}\right)=y_{i}{ }_{2}\left(\theta_{i}\right), z_{i}{ }^{S_{1}}\left(\theta_{i}\right)=z_{i} S_{2}\left(\theta_{i}\right)$, $\forall S_{1}, S_{2} \in \mathcal{S E}$.

Teorema 6: Bajo las hipótesis del Modelo Simétrico inicial con $\$ \mathrm{n} \$$ empresas, se consideran dos modelos de subasta $S_{1}, S_{2} \in \mathcal{S E}$. Sean $P_{i}^{S_{1}}\left(\theta_{i}\right)$ y $P_{i}^{S_{2}}\left(\theta_{i}\right)$ el ingreso total esperado por la empresa $i \in\{1, \ldots, n\}$ en los dos modelos de subasta $S_{1}$ y $S_{2}$, respectivamente. Entonces, si $P_{i}^{S_{1}}\left(a_{2}\right)=P_{i}^{S_{2}}\left(a_{2}\right)$, se verifica $P_{i}^{S_{1}}\left(\theta_{i}\right)=P_{i}^{S_{2}}\left(\theta_{i}\right) \forall \theta_{i} \in\left[a_{1}, a_{2}\right]$.

Demostración: En el apéndice.

Hemos demostrado que si en dos modelos de subasta eléctrica, una empresa $i$ que tiene tipo máximo $a_{2}$, espera ingresar lo mismo en ambos modelos $\left(P_{i}^{S_{1}}\left(a_{2}\right)=P_{i}^{S_{2}}\left(a_{2}\right)\right)$, entonces se verifica que la empresa $i$ espera ingresar lo mismo bajo dichos modelos de subasta, sea cual sea su tipo $\left(P_{i}^{S_{1}}\left(\theta_{i}\right)=P_{i}^{S_{2}}\left(\theta_{i}\right)\right.$, $\left.\forall \theta_{i} \in\left[a_{1}, a_{2}\right]\right)$. Además esto implica que el precio que espera pagar el Operador del Mercado, por la demanda eléctrica, también coincide en ambos modelos

$$
\left(P_{\text {omer }}^{S_{1}}=2 \int_{a_{1}}^{a_{2}} P_{i}^{S_{1}}\left(\theta_{i}\right) f\left(\theta_{i}\right) d \theta_{i}=2 \int_{a_{1}}^{a_{2}} P_{i}^{S_{2}}\left(\theta_{i}\right) f\left(\theta_{i}\right) d \theta_{i}=P_{\text {omer }}^{S_{2}}\right) .
$$

La generalización a modelos de subasta de $m$ objetos homogéneos más uno heterogéneo, en un contexto que no sea el eléctrico, es inmediata.

Podemos obtener, como corolario de este último teorema, la equivalencia de ingresos de cada una de las dos empresas bajo cualquier modelo de subasta $S$ perteneciente al Modelo de Subasta General MSG.

Corolario 7: Supongamos que se verifican las hipótesis del Modelo Simétrico Inicial y sea $S \in \mathrm{MSG}$, entonces las dos empresas esperan el mismo ingreso y el Operador del Mercado espera pagar lo mismo por la demanda eléctrica $D$, independientemente de la elección de $S$. Además el ingreso esperado por las empresas es:

$$
P_{i}^{S}\left(\theta_{i}\right)=\left\{\begin{array}{ccc}
\int_{\theta_{i}}^{a_{2}} g(\alpha, t) f(t) d t & \text { si } & D=\alpha<1 \\
\int_{\theta_{i}}^{a_{2}}(g(1, t)-g(\alpha, t)) f(t) d t+\alpha b_{\max } & \text { si } & 1 \leq D=1+\alpha<2 \\
b_{\max } & \text { si } & D \geq 2
\end{array}\right.
$$

y el pago que espera realizar el Operador del Mercado es 


$$
\begin{aligned}
P_{\text {omer }}^{S} & =2 \int_{a_{1}}^{a_{2}} P_{i}^{S}\left(\theta_{i}\right) f\left(\theta_{i}\right) d \theta_{i} \\
& =\left\{\begin{array}{ccc}
g\left(D, a_{2}\right)-\int_{a_{1}}^{a_{2}} \frac{\partial}{\partial \theta_{i}} g\left(D, \theta_{i}\right) F^{2}\left(\theta_{i}\right) d \theta_{i} & \text { si } & D=\alpha<1 \\
2 \alpha b_{\max }+g\left(1, a_{2}\right)-g\left(\alpha, a_{2}\right) & & \\
-\int_{a_{1}}^{a_{2}} \frac{\partial}{\partial t}(g(1, t)-g(\alpha, t)) F^{2}(t) d t & \text { si } & 1 \leq D=1+\alpha<2 \\
2 b_{\max } & \text { si } & D \geq 2
\end{array}\right.
\end{aligned}
$$

$\forall S \in \mathrm{MSG}$.

Demostración: En el apéndice.

En realidad no es sorprendente este resultado en el Caso 1 y en el Caso 3. El Caso 1 es un caso particular de subasta de un objeto, el lote completo $\alpha$, en el que se verificaba el Teorema de Equivalencia de Ingresos (Myerson, 1981). El Caso 3 es un caso con poca relevancia por ausencia de competencia. Es en realidad el Caso 2 el que establece una generalización novedosa del Teorema de Equivalencia de Ingresos (Myerson, 1981). En él se ha demostrado que, bajo ciertas hipótesis, dos modelos que subastan múltiples objetos son equivalentes en cuanto a los ingresos esperados por los jugadores y pago esperado por el subastador.

\section{Conclusiones}

El aporte principal de este trabajo es la agrupación, en una única expresión continua, de una familia de modelos de subasta que contiene como casos particulares a los modelos clásicos. Dicha expresión ha permitido el cálculo simultáneo de equilibrios Bayesianos de Nash para todos los modelos. Es decir, las empresas generadoras sabrán cual es la oferta óptima para maximizar sus beneficios esperados que deben emplear bajo cualquiera de los modelos pertenecientes a la familia.

Nos hemos centrado en un modelo de mercado simétrico, con idénticas capacidades de producción, costes independientes y empresas neutrales al riego. Bajo estas hipótesis se ha obtenido un resultado de equivalencia de ingresos para todos los modelos de subasta pertenecientes a la familia.

A pesar de la equivalencia de ingresos, hay otros factores que podría hacer que el Operador del Mercado prefiriera un modelo frente a los restantes. Por ejemplo, si el Operador del Mercado fuese averso al riego éste podría preferir un modelo de subasta con la menor varianza posible de los ingresos.

Por otro lado, bajo hipótesis de mercado diferentes, como puede ser que las empresas tengan diferente capacidad de producción, que los tipos fuesen correlacionados, el resultado de equivalencia de ingresos deja de cumplirse y se 
puede encontrar un ranking entre los modelos de subasta pertenecientes a MSG. Es más, bajo determinadas hipótesis puede encontrarse que modelos pertenecientes a la familia MSG no clásicos sean más ventajosos. Veamos un ejemplo:

\subsection{Tipos correlacionados}

Supongamos que hay dos empresas, $i \in\{1,2\}$, con capacidades de producción idénticas $k=1$, siendo $k$ perfectamente divisible que intentan satisfacer una demanda $D=1.5$. Sea la función de coste de la empresa $i, g\left(q_{i}, \theta_{i}\right)=q_{i}, \theta_{i}$. Ahora supondremos que los tipos $\theta_{1}$ y $\theta_{2}$ dependen del precio de la materia prima. Para modelizar la incertidumbre que las empresas tienen sobre él, consideramos una variable aleatoria $S$ que sigue una distribución uniforme en el intervalo [0,1] y las variables $\Theta_{1}\left|S=s, \Theta_{2}\right| S=s$ siguen una distribución uniforme en el intervalo $[s, s+1]$. Las variables aleatorias $\Theta_{i} \mid S=s, i \in\{1,2\}$, son consideradas como variables aleatorias, independientes, indénticamente distribuidas y absolutamente continuas, cuya realización sólo es conocida por la empresa $i$. La función de densidad conjunta de $\theta_{1}, \theta_{2}$ viene dada por:

$$
f_{\left(\Theta_{1}, \Theta_{2}\right)}\left(\theta_{1}, \theta_{2}\right)=\left\{\begin{array}{ccc}
\min \left(\theta_{1}, \theta_{2}\right) & \text { si } & \theta_{1}, \theta_{2} \in[0,1] \\
2-\max \left(\theta_{1}, \theta_{2}\right) & \text { si } & \theta_{1}, \theta_{2} \in[1,2] \\
\min \left(\theta_{1}, \theta_{2}\right) \in[0,1], \\
\max \left(\theta_{1}, \theta_{2}\right) \in[1,2]
\end{array} \text { si } \begin{array}{c}
\max \left(\theta_{1}, \theta_{2}\right)+1 \\
0
\end{array}\right.
$$

A partir de ésta se calcula $f_{\Theta_{j} \mid \Theta_{i}=\theta_{i}}\left(\theta_{j}\right)$ y $F_{\Theta_{j} \mid \Theta_{i}=\theta_{i}}\left(\theta_{j}\right), i, j \in\{1,2\}$ con $i \neq j$, la función de densidad y de distribución, respectivamente, del tipo del jugador $j$ condicionada al conocimiento del tipo del jugador $i$. La conjetura del jugador $i$ sobre el tipo del jugador $j$ procederá del cálculo, mediante la regla de Bayes, de la distribución de probabilidad del tipo del jugador $j$ condicionada al conocimiento del propio tipo, partiendo de la distribución a priori de probabilidad conjunta.

Como la demanda supera la capacidad de producción de una sola de las empresas nos encontramos en el Caso 2 y por tanto la familia de modelos de subasta MSG se reduce a una familia biparamétrica con beneficio:

$$
B_{i}^{\left(\gamma_{1}, \gamma_{2}\right)}\left(\theta_{i}, b_{i}, b_{j}\right)=\left\{\begin{array}{ccc}
\gamma_{1} b_{i}+\left(0.5-\gamma_{1}+\gamma_{2}\right) b_{j}+\left(0.5-\gamma_{2}\right) b_{\max }-\theta_{i} & \text { si } & b_{i}<b_{j} \\
\gamma_{2} b_{i}+\left(0.5-\gamma_{2}\right) b_{\max }-0.5 \theta_{i} & \text { si } & b_{i}>b_{j}
\end{array}\right.
$$

donde $\gamma_{1} \in\left[0,1-\alpha+\gamma_{2}\right]$ y $\gamma_{2} \in[0, \alpha]$. Vamos a comparar los cuatro modelos que se sitúan en los vértices de $\left[0,1-\alpha+\gamma_{2}\right] \times[0, \alpha]$, que son:

- $\quad$ Modelo uniforme $\left(\gamma_{1}=0, \gamma_{2}=0.5\right)$. 
CUADRO 1

EQUILIBRIO BAYESIANO DE NASH E INGRESO ESPERADO

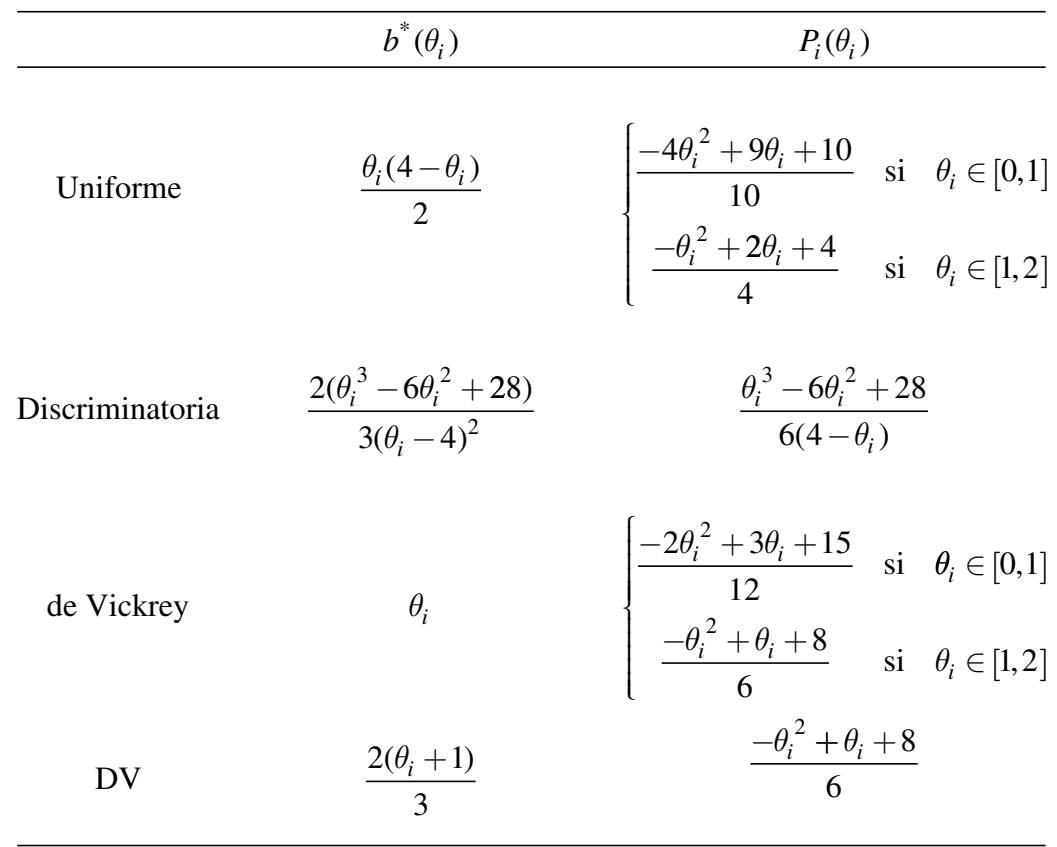

Fuente: Elaboración propia.

FIGURA 1

INGRESOS ESPERADOS POR LA EMPRESA $i$

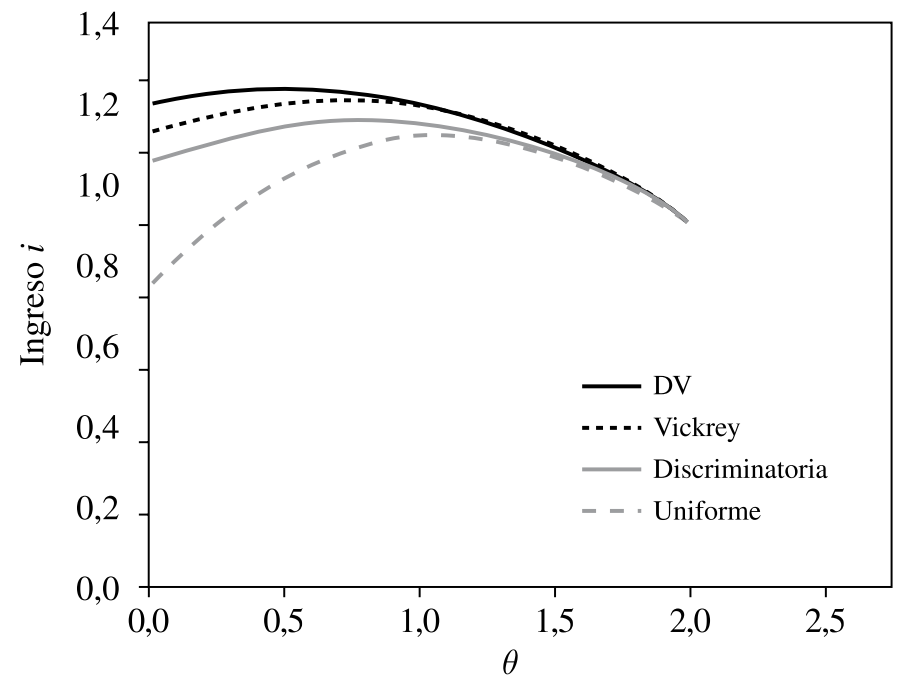

Fuente: Elaboración propia. 
- $\quad$ Modelo discriminatorio $\left(\gamma_{1}=1, \gamma_{2}=0.5\right)$.

- $\quad$ Modelo de Vickrey $\left(\gamma_{1}=0, \gamma_{2}=0\right)$.

En el cuarto vértice se sitúa un modelo de subasta nuevo que llamamos DV:

- $\quad$ Modelo DV $\left(\gamma_{1}=0.5, \gamma_{2}=0\right)$.

Pues bien, es precisamente el modelo DV el modelo el que da mayores ingresos esperados a las empresas. Para comprobarlo se procede de la misma manera que a lo largo del artículo, se calculan los equilibrios Bayesianos de Nash para cada uno de los modelos (teniendo en cuenta, por supuesto la nueva relación entre los tipos) y a continuación se calcula el ingreso esperado por cada una de las empresas que viene dado por:

$$
\begin{aligned}
P_{i}^{\left(\gamma_{1}, \gamma_{2}\right)}\left(\theta_{i}\right)= & \left(\gamma_{1}-\left(\gamma_{1}-\gamma_{2}\right) F_{\Theta_{j} \mid \Theta_{i}=\theta_{i}}\left(\theta_{i}\right)\right) b^{*}\left(\theta_{i}\right)+2\left(0.5-\gamma_{2}\right) \\
& +\left(0.5-\gamma_{1}+\gamma_{2}\right) \int_{\theta_{i}}^{2} b^{*}(t) f_{\Theta_{j} \mid \Theta_{i}=\theta_{i}}(t) d t
\end{aligned}
$$

OBSERVACIÓN 8: En general, el ingreso esperado por las empresas, si éstas juegan el equilibrio correspondiente, no coincide en todos los modelos de subasta. Sin embargo, se comprueba fácilmente que si $F_{\Theta_{j} \mid \Theta_{i}=\theta_{i}}\left(\theta_{j}\right)=F_{\Theta_{j}}\left(\theta_{j}\right)$ y $f_{\Theta_{j} \mid \Theta_{i}=\theta_{i}}\left(\theta_{j}\right)=f_{\Theta_{j}}\left(\theta_{j}\right)$ (es decir, si los tipos fuesen independientes) entonces, la expresión del ingreso esperado por la empresa $i$ queda como en Corolario 7 para el caso 2, es decir independiente de los parámetros y verificándose un resultado de equivalencia de ingreso.

El Cuadro 1 muestra el equilibrio Bayesiano de Nash y el ingreso esperado por las empresas en cada uno de los cuatro modelos mencionados.

En la Figura 1 se ha representado los ingresos esperados por las empresas bajo los cuatro modelos de subasta, donde se observa que efectivamente el modelo que da mayores ingresos esperados a las empresas es el modelo DV.

Todos los resultados obtenidos en el trabajo son fácilmente generalizados a modelos de subasta en un contexto no eléctrico. Por ejemplo si dos compradores de obras de arte verificasen las hipótesis del último ejemplo (sus valoraciones dependen de un valor común de esas obras en el mercado), tendrían menores pagos esperados en aquella sala de arte que utilizase un modelo DV para subastar sus obras. 


\section{REFERENCIAS}

Ausubel, L. and P. Cramton (2002), "Demand Reduction and Inefficiency in Multi-Unit Auctions.” Technical Report 96-07. Department of Economics, University of Maryland.

Bosco, B. and L. Parisio (2001), "Market Power and The Power Market: Multiunit Bidding and (In)efficiency of the Italian Electricity Market." Quaderni ref. $\mathrm{N}^{\circ} 6 /$ Ottobre 2001. Ricerche e Consulenze per l'Economia e la Finanza.

Burguet, R. (2000), “Auction Theory: A Guided Tour.” Investigaciones Económicas 24, pp. 3-50.

Fabra, N. (2001), "Market Power in Electricity Markets.” Thesis Department of Economics, European University Institute.

Fabra, N.; N. Fehr and D. Harbord (2002), "Designing Electricity Auctions: Uniform, Discriminatory and Vickrey.” Harvard Electricity Policy Group Reseach Paper.

Fabra, N.; N. Fehr and D. Harbord (2006), "Designing Electricity Auctions." The RAND Journal of Economics 37(1), pp 23-46.

Federico, G. and D. Rahman (2001), "Bidding in an Electrity Pay-As-Bid Auction." Journal of Regulatory Economics 24(2), pp. 175-211.

Fehr, N.H. and D. Harbord (1993), "Spot Market Competition in the UK

Electricity Industry." The Economic Journal, 103, pp. 531-546.

Fehr, N.H. and D. Harbord (1998), "Competition in Electricity Spot Markets: Economic Theory and International Experience.” University of Oslo Memorandum in Economics; $\mathrm{N}^{\circ}$.

Ferrero, R.W., J.F. Rivera and S.M. Shahidehpour (1998), "Application of Games with Incomplete Information for Pricing Electricity in Deregulated Power Pools." IEEE Transactions on Power Systems 13(1), February 1998.

Hinz, J. (2004), "A revenue equivalence theorem for electricity auctions. Journal of Applied Probability 41, pp. 299-314.

Klemperer, P.(1999), “Auction Theory: A Guide to the Literature.” Journal of Economic Surveys, 13.

Krishna, V. (2002), Auction Theory. Elsevier Academic Press, pag. 200.

Milgrom, P. (2004). Putting Auction Theory to Work. Cambridge University Press.

Myerson, R. (1981), “Optimal Auction Design.” Mathematics of Operations Research", 6, pp. 58-73.

Schöne, S. (2003), "Capacity Constraint and Stochastic Costs: A Multi-Unit Auction in the Electricity Spot Market.” Discussion Papers in Business, $\mathrm{N}^{\circ} 30$. Humboldt-Universitl" $\{a\} t$ zu Berlin.

Simmons, F. (1990). Ecuaciones Diferenciales con aplicaciones y notas históricas. Mc Graw Hill.

Vickrey, W. (1961), "Counterspeculation, Auctions and Competitive Sealed Tenders." Journal of Finance, 16, pp. 8-37.

Wolfram, C. (1999). "Electricity Markets: Should the Rest of the World Adopt the UK Reforms?." Regulation, 22, pp. 48-53. 


\section{ApÉNDICE}

\section{Demostración de la Proposición 1}

La empresa $i$ conoce su propio tipo $\theta_{i}$, pero $\theta_{j}$ es una variable aleatoria para ella. El beneficio de la empresa $i$ viene dado por:

$$
B_{i}\left(\theta_{i}, b_{i}, b\left(\theta_{j}\right)\right)=\left\{\begin{array}{ccc}
\gamma_{1} b_{i}+\beta_{1} b\left(\theta_{j}\right)+\varphi b_{\max }-g\left(\phi_{1}, \theta_{i}\right) & \text { si } & b^{-1}\left(b_{i}\right)<\theta_{j} \\
\gamma_{2} b_{i}+\varphi b_{\max }-g\left(\phi_{2}, \theta_{i}\right) & \text { si } & b^{-1}\left(b_{i}\right)>\theta_{j}
\end{array}\right.
$$

El beneficio esperado por la empresa $i$, dado que su tipo es $\theta_{i}$, es:

$$
B M_{i}\left(\theta_{i}, b_{i}, b\right)=\int_{a_{1}}^{a_{2}} B_{i}\left(\theta_{i}, b_{i}, b\left(\theta_{j}\right)\right) f\left(\theta_{j} \mid \theta_{i}\right) d \theta_{j}
$$

Teniendo en cuenta que los tipos son variables aleatorias independientes:

$$
\begin{aligned}
B M_{i}\left(\theta_{i}, b_{i}, b\right) & =\int_{a_{1}}^{a_{2}} B_{i}\left(\theta_{i}, b_{i}, b\left(\theta_{j}\right)\right) f\left(\theta_{j}\right) d \theta_{j} \\
& =\int_{a_{1}}^{b^{-1}\left(b_{i}\right)}\left(\gamma_{2} b_{i}+\varphi b_{\max }-g\left(\phi_{2}, \theta_{i}\right)\right) f\left(\theta_{j}\right) d \theta_{j} \\
& +\int_{b^{-1}\left(b_{i}\right)}^{a_{2}}\left(\gamma_{1} b_{i}+\beta_{1} b\left(\theta_{j}\right)+\varphi b_{\max }-g\left(\phi_{1}, \theta_{i}\right)\right) f\left(\theta_{j}\right) d \theta_{j} .
\end{aligned}
$$

Entonces, $b_{i}$ es la mejor respuesta de la empresa $i$ si maximiza el beneficio esperado, dado que su tipo es $\theta_{i}$. Derivando respecto de $b_{i}$ se tiene, aplicando el Teorema Fundamental del Cálculo:

$$
\begin{aligned}
\frac{\partial}{\partial b_{i}} B M_{i}\left(\theta_{i}, b_{i}, b\right)= & \left(\gamma_{2}-\gamma_{1}\right) F\left(b^{-1}\left(b_{i}\right)\right)+\gamma_{1} \\
& +\left(-\left(\phi_{1}-\phi_{2}\right) b_{i}+g\left(\phi_{1}, \theta_{i}\right)-g\left(\phi_{2}, \theta_{i}\right)\right) f\left(b^{-1}\left(b_{i}\right)\right) \frac{d}{d b_{i}}\left(b^{-1}\left(b_{i}\right)\right) .
\end{aligned}
$$

Como $b^{-1}\left(b_{i}\right)=\theta_{i} \Leftrightarrow b_{i}=b\left(\theta_{i}\right)$, reemplazando e igualando a cero, obtenemos la siguiente ecuación diferencial:

$$
\begin{aligned}
& \left(\left(\gamma_{2}-\gamma_{1}\right) F\left(\theta_{i}\right)+\gamma_{1}\right) b^{* \prime}\left(\theta_{i}\right)-\left(\phi_{1}-\phi_{2}\right) f\left(\theta_{i}\right) b^{*}\left(\theta_{i}\right) \\
= & -\left(g\left(\phi_{1}, \theta_{i}\right)-g\left(\phi_{2}, \theta_{i}\right)\right)\left(\theta_{i}\right) .
\end{aligned}
$$


Luego, todo equilibrio Bayesiano de Nash simétrico es solución de la ecuación diferencial ordinaria anterior. La ecuación diferencial con la condición inicial $b^{*}\left(a_{2}\right)=b_{\max }$ forma un problema de valor inicial que verifica las hipótesis de una extensión del teorema de existencia y unicidad de Picard (Simmons, 1990, pag.485-486) para ecuaciones diferenciales ordinarias lineales, siempre y cuando $\left(\gamma_{2}-\gamma_{1}\right) F\left(\theta_{i}\right)+\gamma_{1}$ sea distinto de cero en $a_{2}$. Se obtienen así los diferentes casos que pasamos a describir:

Región I: Si $\gamma_{2} \neq 0$ se verifica que $\left(\left(\gamma_{2}-\gamma_{1}\right) F\left(a_{2}\right)+\gamma_{1}\right)=\gamma_{2} \neq 0$ y cumple la condición anterior. Por tanto, existe un único equilibrio Bayesiano de Nash simétrico $\left(b^{*}\left(\theta_{1}\right), b^{*}\left(\theta_{2}\right)\right)$ que cumple $b^{*}\left(a_{2}\right)=b_{\max }$ que se obtiene integrando la ecuación diferencial anterior:

$$
\begin{aligned}
b^{*}\left(\theta_{i}\right)= & \frac{g\left(\phi_{1}, \theta_{i}\right)-g\left(\phi_{2}, \theta_{i}\right)}{\phi_{1}-\phi_{2}}+\left(\gamma_{1}-\left(\gamma_{1}-\gamma_{2}\right) F\left(\theta_{i}\right)\right)^{-\frac{\phi_{1}-\phi_{2}}{\gamma_{1}-\gamma_{2}}}\left(b_{\max } \gamma_{2}^{\frac{\phi_{1}-\phi_{2}}{\gamma_{1}-\gamma_{2}}}+\right. \\
& \frac{1}{\phi_{1}-\phi_{2}} \int_{\theta_{i}}^{a_{2}} \frac{\partial}{\partial t}\left(g\left(\phi_{1}, t\right)-g\left(\phi_{2}, t\right)\right)\left(\gamma_{1}-\left(\gamma_{1}-\gamma_{2}\right) F(t)\right)^{\frac{\phi_{1}-\phi_{2}}{\gamma_{1}-\gamma_{2}}} d t \\
& \left.-\frac{g\left(\phi_{1}, a_{2}\right)-g\left(\phi_{2}, a_{2}\right)}{\phi_{1}-\phi_{2}} \gamma_{2}^{\frac{\phi_{1}-\phi_{2}}{\gamma_{1}-\gamma_{2}}}\right)
\end{aligned}
$$

si $\gamma_{1} \neq \gamma_{2}, \mathrm{y}$

$$
\begin{aligned}
b^{*}\left(\theta_{i}\right)= & \frac{g\left(\phi_{1}, \theta_{i}\right)-g\left(\phi_{2}, \theta_{i}\right)}{\phi_{1}-\phi_{2}}+e^{-\frac{\phi_{1}-\phi_{2}}{\gamma_{1}}\left(1-F\left(\theta_{i}\right)\right)}\left(b_{\max }-\frac{g\left(\phi_{1}, a_{2}\right)-g\left(\phi_{2}, a_{2}\right)}{\phi_{1}-\phi_{2}}\right) \\
& +\frac{e^{\frac{\phi_{1}-\phi_{2}}{\gamma_{1}}\left(1-F\left(\theta_{i}\right)\right)}}{\phi_{1}-\phi_{2}} \int_{\theta_{i}}^{a_{2}} \frac{\partial}{\partial t}\left(g\left(\phi_{1}, t\right)-g\left(\phi_{2}, t\right)\right) e^{-\frac{\phi_{1}-\phi_{2}}{\gamma_{1}}(1-F(t))} d t
\end{aligned}
$$

si $\gamma_{1}=\gamma_{2}$.

Región II: Si $\gamma_{2}=0$ y $\gamma_{1}=0$, se verifica $\left(\left(\gamma_{2}-\gamma_{1}\right) F\left(\theta_{i}\right)+\gamma_{1}\right)=0, \forall \theta_{i} \in\left[a_{1}, a_{2}\right]$ y la ecuación diferencial se reduce a:

$$
b^{*}\left(\theta_{i}\right)=\frac{g\left(\phi_{1}, \theta_{i}\right)-g\left(\phi_{2}, \theta_{i}\right)}{\phi_{1}-\phi_{2}}
$$

que es estrategia dominante para $i$ ya que $\partial B M_{i}\left(\theta_{i}, b_{i}, b\right) / \partial b_{i}$ no depende de $b$. Región III: Si $\gamma_{2}=0$ y $\gamma_{1} \neq 0$, se verifica que:

$$
\gamma_{1}\left(1-F\left(\theta_{i}\right) b^{*^{\prime}}\left(\theta_{i}\right)-\left(\phi_{1}-\phi_{2}\right) f\left(\theta_{i}\right) b^{*}\left(\theta_{i}\right)=-\left(g\left(\phi_{1}, \theta_{i}\right)-g\left(\phi_{2}, \theta_{i}\right)\right) f\left(\theta_{i}\right) .\right.
$$


Si evaluamos la expresión anterior en $\theta_{i}=a_{2}$ queda que todo equilibrio Bayesiano de Nash simétrico cumple:

$$
b^{*}\left(a_{2}\right)=\frac{g\left(\phi_{1}, a_{2}\right)-g\left(\phi_{2}, a_{2}\right)}{\phi_{1}-\phi_{2}} .
$$

La solución general de la ecuación diferencial es, integrando:

$$
\begin{aligned}
b^{*}\left(\theta_{i}\right)= & \frac{g\left(\phi_{1}, \theta_{i}\right)-g\left(\phi_{2}, \theta_{i}\right)}{\phi_{1}-\phi_{2}}+ \\
& \left(1-F\left(\theta_{i}\right)\right)^{-\frac{\phi_{1}-\phi_{2}}{\gamma_{1}}}\left(\frac{1}{\phi_{1}-\phi_{2}} \int \frac{\partial}{\partial \theta_{i}}\left(g\left(\phi_{1}, \theta_{i}\right)-g\left(\phi_{2}, \theta_{i}\right)\right)\left(1-F\left(\theta_{i}\right)\right)^{\frac{\phi_{1}-\phi_{2}}{\gamma_{1}}} d \theta_{i}+C\right) .
\end{aligned}
$$

Como:

$$
\begin{aligned}
\lim _{\theta_{i} \rightarrow a_{2}^{-}} b^{*}\left(\theta_{i}\right)= & \frac{g\left(\phi_{1}, a_{2}\right)-g\left(\phi_{2}, a_{2}\right)}{\phi_{1}-\phi_{2}}+ \\
& \frac{\frac{1}{\phi_{1}-\phi_{2}} \int \frac{\partial}{\partial \theta_{i}}\left(g\left(\phi_{1}, \theta_{i}\right)-g\left(\phi_{2}, \theta_{i}\right)\right)\left(1-F\left(\theta_{i}\right)\right)^{\frac{\phi_{1}-\phi_{2}}{\gamma_{1}}} d \theta_{i}+C}{\left(1-F\left(\theta_{i}\right)\right)^{\frac{\phi_{1}-\phi_{2}}{\gamma_{1}}}}
\end{aligned}
$$

para que este último límite sea finito, teniendo en cuenta que $\left(\phi_{1}-\phi_{2}\right) / \gamma_{1}>0$, la constante debe verificar:

$$
C=-\frac{1}{\phi_{1}-\phi_{2}}\left[\int \frac{\partial}{\partial t}\left(g\left(\phi_{1}, t\right)-g\left(\phi_{2}, t\right)\right) 1-F(t)^{\frac{\phi_{1}-\phi_{2}}{\gamma_{1}}} d t\right]_{t=a_{2}}
$$

Luego, sustituyendo el valor de la constante, la solución particular de la ecuación diferencial queda:

$$
\begin{aligned}
b^{*}\left(\theta_{i}\right)= & \frac{g\left(\phi_{1}, \theta_{i}\right)-g\left(\phi_{2}, \theta_{i}\right)}{\phi_{1}-\phi_{2}} \\
& +\frac{\left(1-F\left(\theta_{i}\right)\right)^{-\frac{\phi_{1}-\phi_{2}}{\gamma_{1}}}}{\phi_{1}-\phi_{2}} \int_{\theta_{i}}^{a_{2}} \frac{\partial}{\partial t}\left(g\left(\phi_{1}, t\right)-g\left(\phi_{2}, t\right)\right)(1-F(t))^{\frac{\phi_{1}-\phi_{2}}{\gamma_{1}}} d t .
\end{aligned}
$$




\section{Demostración Corolario 3}

En efecto, si tomamos $b^{*}\left(a_{2}\right)=\left(g\left(\phi_{1}, a_{2}\right)-g\left(\phi_{2}, a_{2}\right)\right) /\left(\phi_{1}-\phi_{2}\right)$ en las expresiones del equilibrio Bayesiano de Nash obtenida en la Proposición 1 para la Región I, se obtienen las expresiones del enunciado.

\section{Demostración Teorema 6}

Dado un modelo de subasta $S \in \mathcal{S E}$ con regla de asignación

$$
\left\{\omega_{i}^{S}\left(b_{1}, \ldots, b_{n}\right), \rho_{i}^{S}\left(b_{1}, \ldots, b_{n}\right), \psi_{i}^{S}\left(b_{1}, \ldots, b_{n}\right), \chi_{i}^{S}\left(b_{1}, \ldots, b_{n}\right)\right\}_{i=1}^{n}
$$

y único equilibrio Bayesiano de Nash $\left(b^{*}\left(\theta_{1}\right), \ldots, b^{*}\left(\theta_{n}\right)\right)$, si consideramos el mecanismo directo $\tilde{S}$ asociado a $S$ con regla de asignación

$$
\begin{aligned}
& \left\{\omega_{i}^{\tilde{S}}\left(t_{1}, \ldots, t_{n}\right), \rho_{i}^{\tilde{S}}\left(t_{1}, \ldots, t_{n}\right), \psi_{i}^{\tilde{S}}\left(t_{1}, \ldots, t_{n}\right), \chi_{i}^{\tilde{S}}\left(t_{1}, \ldots, t_{n}\right)\right\}_{i=1}^{n} \\
& =\left\{\tilde{\omega}_{i}\left(t_{1}, \ldots, t_{n}\right), \tilde{\rho}_{i}\left(t_{1}, \ldots, t_{n}\right), \tilde{\psi}_{i}\left(t_{1}, \ldots, t_{n}\right), \tilde{\chi}_{i}\left(t_{1}, \ldots, t_{n}\right)\right\}_{i=1}^{n},
\end{aligned}
$$

por el Principio de Revelación este modelo $\tilde{S}$ es de incentivos compatibles.

Luego, el beneficio esperado por un jugador $i$, si su declaración es $t_{i}$ y el resto de jugadores confiesan el verdadero tipo, bajo el modelo $\tilde{S}$ viene dado por:

$$
\begin{aligned}
B M_{i}^{\tilde{S}}\left(t_{i}\right)= & -\int_{\Theta_{-i}} g\left(\alpha, \theta_{i}\right) \psi_{i}^{\tilde{S}}\left(\theta_{-i}, t_{i}\right) f_{-i}\left(\theta_{-i}\right) d \theta_{-i} \\
& -\int_{\Theta_{-i}} g\left(1, \theta_{i}\right) \chi_{i}^{\tilde{S}}\left(\theta_{-i}, t_{i}\right) f_{-i}\left(\theta_{-i}\right) d \theta_{-i} \\
& +\int_{\Theta_{-i}} \omega_{i}^{\tilde{S}}\left(\theta_{-i}, t_{i}\right) \psi_{i}^{\tilde{S}}\left(\theta_{-i}, t_{i}\right) f_{-i}\left(\theta_{-i}\right) d \theta_{-i} \\
& +\int_{\Theta_{-i}} \rho_{i}^{\tilde{S}}\left(\theta_{-i}, t_{i}\right) \chi_{i}^{\tilde{S}}\left(\theta_{-i}, t_{i}\right) f_{-i}\left(\theta_{-i}\right) d \theta_{-i} \\
& =q_{i}^{S}\left(t_{i}\right)+p_{i}^{S}\left(t_{i}\right)-g\left(\alpha, \theta_{i}\right) y_{i}^{S}\left(t_{i}\right)-g\left(1, \theta_{i}\right) z_{i}^{S}\left(t_{i}\right),
\end{aligned}
$$

Como los jugadores actúan de tal manera que maximicen su beneficio, usarán las estrategias en equilibrio y, por el Principio de Revelación, obtenemos:

$\theta_{i}=\arg \max _{t_{i}}\left\{q_{i}^{S}\left(t_{i}\right)+p_{i}^{S}\left(t_{i}\right)-g\left(\alpha, \theta_{i}\right) y_{i}^{S}\left(t_{i}\right)-g\left(1, \theta_{i}\right) z_{i}^{S}\left(t_{i}\right)\right\}$

quedando la ecuación:

$\left(q_{i}^{S}\right)^{\prime}\left(\theta_{i}\right)+\left(p_{i}^{S}\right)^{\prime}\left(\theta_{i}\right)=g\left(\alpha, \theta_{i}\right)\left(y_{i}^{S}\left(\theta_{i}\right)\right)^{\prime}+g\left(1, \theta_{i}\right)\left(z_{i}^{S}\left(\theta_{i}\right)\right)^{\prime}$. 
Integrando ambos lados de la igualdad se obtiene:

$$
\begin{aligned}
& q_{i}^{S}\left(\theta_{i}\right)+p_{i}^{S}\left(\theta_{i}\right) \\
&=-\int_{\theta_{i}}^{a_{2}} g(1, t)\left(z_{i}^{S}\right)^{\prime}(t) d t-\int_{\theta_{i}}^{a_{2}} g(\alpha, t)\left(y_{i}^{S}\right)^{\prime}(t) d t+q_{i}^{S}\left(a_{2}\right)+p_{i}^{S}\left(a_{2}\right) \\
& P_{i}^{S}\left(\theta_{i}\right)=-\int_{\theta_{i}}^{a_{2}} g(1, t)\left(z_{i}^{S}\right)^{\prime}(t) d t-\int_{\theta_{i}}^{a_{2}} g(\alpha, t)\left(y_{i}^{S}\right)^{\prime}(t) d t+P_{i}^{S}\left(a_{2}\right)
\end{aligned}
$$

Como $y_{i}^{S_{1}}\left(\theta_{i}\right)=y_{i}^{S_{2}}\left(\theta_{i}\right), z_{i}^{S_{1}}\left(\theta_{i}\right)=z_{i}^{S_{2}}\left(\theta_{i}\right) \forall \theta_{i} \in\left[a_{1}, a_{2}\right]$ y $\forall S_{1}, S_{2} \in \mathcal{S E}$ y, por el enunciado, $P_{i}^{S_{1}}\left(a_{2}\right)=P_{i}^{S_{2}}\left(a_{2}\right)$ entonces $P_{i}^{S_{1}}\left(\theta_{i}\right)=P_{i}^{S_{2}}\left(\theta_{i}\right) \forall \theta_{i} \in\left[a_{1}, a_{2}\right]$.

\section{Demostración Corolario 7}

Obviamente $\mathrm{MSG} \subset \mathcal{S E}$ ya que:

$$
\begin{aligned}
M S G= & \left\{S \in \mathcal { S E } \text { tales que } n = 2 \text { y } \left\{\rho_{i}^{S}\left(b_{1}, b_{2}\right)=\gamma_{1} b_{i}+\beta_{1} b_{j}+\varphi b_{\max },\right.\right. \\
& \left.\left.\omega_{i}^{S}\left(b_{1}, b_{2}\right)=\gamma_{2} b_{i}+\varphi b_{\max }\right\}\right\} .
\end{aligned}
$$

Vamos a aplicar el teorema anterior con $n=2$ en las tres situaciones del tamaño de la demanda, tanto para demostrar que $P_{i}{ }_{i}\left(\theta_{i}\right)$ no depende del modelo $S$ considerado, como para el cálculo del ingreso esperado por las empresas.

Caso $1(D=\alpha \leq 1)$.

Este caso corresponde al valor $m=0 \mathrm{y}$, en él, la regla de asignación de un modelo cualquiera $S \in M S G$ es:

$$
\begin{aligned}
\rho_{i}^{S}\left(b_{1}, b_{2}\right) & =\gamma_{1} b_{i}+\left(\alpha-\gamma_{1}\right) b_{j} \\
\omega_{i}^{S}\left(b_{1}, b_{2}\right) & =0 \\
\psi_{i}^{S}\left(b_{1}, b_{2}\right) & =\operatorname{Prob}\left(b_{i}<b_{j}\right) \\
\chi_{i}^{S}\left(b_{1}, b_{2}\right) & =0 .
\end{aligned}
$$

\section{Luego}

$$
\begin{aligned}
& \tilde{\psi}_{i}\left(\theta_{1}, \theta_{2}\right)=\operatorname{Prob}\left(\theta_{i}<\theta_{j}\right) \\
& \tilde{\chi}_{i}\left(\theta_{1}, \theta_{2}\right)=0,
\end{aligned}
$$


y se obtiene

$$
\begin{aligned}
& y_{i}^{S}\left(\theta_{i}\right)=1-F\left(\theta_{i}\right) \\
& z_{i}^{S}\left(\theta_{i}\right)=0 .
\end{aligned}
$$

Si $\theta_{i}=a_{2}$, la empresa $i$ queda fuera del mercado y en cualquiera de los modelos $S \in \mathrm{MSG}$ se verifica que $P_{i} S_{(}\left(a_{2}\right)=0$. Luego, por el teorema anterior se tiene que $P_{i} S_{(}\left(\theta_{i}\right) \forall \theta_{i} \in\left[a_{1}, a_{2}\right]$ es el mismo $\forall S \in \mathrm{MSG}$, es decir el mismo ingreso esperado bajo todos los modelos considerados.

El ingreso esperado por la empresa $i$ bajo cualquier modelo $S \in \mathrm{MSG}$ es:

$$
P_{i}^{S}\left(\theta_{i}\right)=-\int_{\theta_{i}}^{a_{2}} g(\alpha, t)\left(y_{i}^{S}\right)^{\prime}(t) d t=\int_{\theta_{i}}^{a_{2}} g(\alpha, t) f(t) d t
$$

Caso $2(1<D=1+\alpha<2)$.

Este caso corresponde al valor $m=1 \mathrm{y}$, en él, la regla de asignación en cada modelo $S \in \mathrm{MSG}$ es:

$$
\begin{aligned}
& \rho_{i}^{S}\left(b_{1}, b_{2}\right)=\gamma_{1} b_{i}+\left(1-\alpha-\gamma_{1}+\gamma_{2}\right) b_{j}+\left(\alpha-\gamma_{2}\right) b_{\max } \\
& \omega_{i}^{S}\left(b_{1}, b_{2}\right)=\gamma_{2} b_{i}+\left(\alpha-\gamma_{2}\right) b_{\max } \\
& \psi_{i}^{S}\left(b_{1}, b_{2}\right)=\operatorname{Prob}\left(b_{i}>b_{j}\right) \\
& \chi_{i}^{S}\left(b_{1}, b_{2}\right)=\operatorname{Prob}\left(b_{i}<b_{j}\right) .
\end{aligned}
$$

Luego:

$$
\begin{aligned}
& \tilde{\psi}_{i}\left(\theta_{1}, \theta_{2}\right)=\operatorname{Prob}\left(\theta_{i}>\theta_{j}\right) \\
& \tilde{\chi}_{i}\left(\theta_{1}, \theta_{2}\right)=\operatorname{Prob}\left(\theta_{i}<\theta_{j}\right),
\end{aligned}
$$

y se obtiene:

$$
\begin{aligned}
& y_{i}^{S}\left(\theta_{i}\right)=F\left(\theta_{i}\right) \\
& z_{i}^{S}\left(\theta_{i}\right)=1-F\left(\theta_{i}\right) .
\end{aligned}
$$

Si $\theta_{i}=a_{2}$, la empresa $i$ despacha la demanda residual y en cualquiera de los modelos $S \in$ MSG se verifica que $P_{i}^{S}\left(a_{2}\right)=\alpha b_{\text {max }}$. Luego, por el teorema anterior se tiene que $P_{i} S_{(}\left(\theta_{i}\right)$ es siempre el mismo $\forall S \in \mathrm{MSG}$, es decir, el mismo ingreso esperado.

El ingreso esperado bajo cualquier modelo $S \in \mathrm{MSG}$ es: 


$$
\begin{aligned}
P_{i}^{S}\left(\theta_{i}\right) & =-\int_{\theta_{i}}^{a_{2}} g(1, t)(1-F(t))^{\prime} d t-\int_{\theta_{i}}^{a_{2}} g(\alpha, t) F(t)^{\prime} d t+\alpha b_{\max } \\
& =\int_{\theta_{i}}^{a_{2}}(g(1, t)-g(\alpha, t)) f(t) d t+\alpha b_{\max } .
\end{aligned}
$$

Caso $3(D \geq 2)$.

Este caso corresponde a los valores $m \in\{2,3, \ldots\}$ y, en él, no hay competencia, verificándose:

$$
\begin{aligned}
P_{i}^{S}\left(a_{2}\right) & =b_{\max } \\
y_{i}^{S}\left(\theta_{i}\right) & =0 \\
z_{i}^{S}\left(\theta_{i}\right) & =1
\end{aligned}
$$

Luego, por el teorema anterior se tiene que $P_{i}^{S}\left(\theta_{i}\right)$ es siempre el mismo bajo todos los modelos $S \in$ MSG. 